\title{
SOME FORMULAS OF RAMANUJAN INVOLVING BESSEL FUNCTIONS
}

\author{
by
}

Henri Cohen

\begin{abstract}
We generalize a number of summation formulas involving the $K$-Bessel function, due to Ramanujan, Koshliakov, and others.

Résumé. - Nous généralisons des formules sommatoires faisant intervenir les fonctions $K$ de Bessel, et qui sont dues à Ramanujan, Koshliakov et d'autres.
\end{abstract}

\section{Introduction and Tools}

In his notebooks, Ramanujan has given a number of interesting summation formulas involving the $K$-Bessel function. These were generalized by Koshliakov and Berndt-Lee-Sohn in [1]. The purpose of the present paper is to give a general framework for these formulas, and to generalize them. We only give a bare sketch of the proofs.

First, we recall that the $K$-Bessel function $K_{\nu}(x)$ with $\nu \in \mathbb{C}$ and $x \in \mathbb{R}_{>0}$ can be defined by the formula

$$
K_{\nu}(x)=\frac{1}{2} \int_{0}^{\infty} t^{\nu-1} e^{-(x / 2)(t+1 / t)} d t
$$

Since $K_{-\nu}=K_{\nu}$, we will always assume implicitly that $\operatorname{Re}(\nu) \geq 0$. These functions possess many important properties, but the most important for us will be their behavior as $x \rightarrow \infty$, and to a lesser extent as $x \rightarrow 0$ : as $x \rightarrow \infty$ we have

$$
K_{\nu}(x) \sim\left(\frac{\pi}{2 x}\right)^{1 / 2} e^{-x}
$$

(note that the right hand side is independent of $\nu$ ), and as $x \rightarrow 0$ we have $K_{\nu}(x) \sim$ $2^{-\nu-1} \Gamma(\nu) x^{-\nu}$ when $\operatorname{Re}(\nu)>0$, and $K_{0}(x) \sim-\log (x)$.

Second, we recall that the Riemann zeta function $\zeta(s)$ which is usually defined by the series $\sum_{n \geq 1} n^{-s}$ for $\operatorname{Re}(s)>1$, can be extended to the whole complex plane into a meromorphic function having a single pole, simple, at $s=1$, and satisfies the functional equation $\Lambda(1-s)=$ $\Lambda(s)$, with $\Lambda(s)=\pi^{-s / 2} \Gamma(s / 2) \zeta(s)$.

Third, we recall that $\sigma_{s}(n)$ denotes the sum of the $s$ th powers of the positive divisors of $n$ : $\sigma_{s}(n)=\sum_{d \mid n} d^{s}$. 
Fourth, we recall that the real dilogarithm function $\operatorname{Li}_{2}(x)$ is defined by $\operatorname{Li}_{2}(x)=-\int_{0}^{x} \log (\mid 1-$ $t \mid) / t d t$, so that $\operatorname{Li}_{2}(x)=\sum_{n \geq 1} x^{n} / n^{2}$ when $|x| \leq 1$. This function has many functional equations, and in particular $\mathrm{Li}_{2}(x)+\mathrm{Li}_{2}(1 / x)=C(x) \pi^{2} / 6-\log ^{2}(x) / 2$, where $C(x)=2$ for $x>0$ and $C(x)=-1$ for $x<0$, which immediately implies the behavior of $\operatorname{Li}_{2}(x)$ when $x \rightarrow \infty$.

Fifth, we define the standard nonholomorphic Eisenstein Series $G(\tau, s)$ for $\operatorname{Re}(s)>1$ and $\operatorname{Im}(\tau)>0$ by

$$
G(\tau, s)=\frac{1}{2} \sum_{(m, n) \in \mathbb{Z}^{2}}^{\prime} \frac{y^{s}}{|m \tau+n|^{2 s}},
$$

where $\sum^{\prime}$ means that the term $(m, n)=(0,0)$ must be omitted, and $\tau=x+i y$. This function is invariant (in $\tau$ ) under the usual action of $\mathrm{SL}_{2}(\mathbb{Z})$, in other words it is a nonholomorphic modular function of weight 0 . It is easy to compute its Fourier expansion, which is given by

$$
\begin{aligned}
G(\tau, s)= & \zeta(2 s) y^{s}+\frac{\pi^{1 / 2} \Gamma(s-1 / 2)}{\Gamma(s)} \zeta(2 s-1) y^{1-s} \\
& +4 y^{1 / 2} \frac{\pi^{s}}{\Gamma(s)} \sum_{n \geq 1} \frac{\sigma_{2 s-1}(n)}{n^{s-1 / 2}} K_{s-1 / 2}(2 \pi n y) \cos (2 \pi n x)
\end{aligned}
$$

In particular this expansion shows that $G(\tau, s)$ has a meromorphic continuation to the whole complex $s$-plane, with a single pole, at $s=1$, which is simple with residue $\pi / 2$ (note that this is independent of $\tau$ ), and if we set

$$
\mathcal{G}(\tau, s)=\pi^{-s} \Gamma(s) G(\tau, s)
$$

we have the functional equation $\mathcal{G}(\tau, 1-s)=\mathcal{G}(\tau, s)$.

\section{The First Theorem and Specializations}

We implicitly consider that variables such as $x, b$, and so forth are real and strictly positive, in particular different from 0.

Theorem 2.1. - We have

$$
\begin{aligned}
& \Lambda(s)\left(x^{(1-s) / 2}-x^{(s-1) / 2}\right)+4 x^{1 / 2} \sum_{n \geq 1} \frac{\sigma_{s}(n)}{n^{s / 2}} K_{s / 2}(2 \pi n x) \\
= & \Lambda(-s)\left(x^{-(1+s) / 2}-x^{(1+s) / 2}\right)+4 x^{-1 / 2} \sum_{n \geq 1} \frac{\sigma_{s}(n)}{n^{s / 2}} K_{s / 2}\left(\frac{2 \pi n}{x}\right) .
\end{aligned}
$$

This immediately follows from the Fourier expansion of $G(\tau, s)$.

Corollary 2.2. -

$$
\begin{aligned}
4 \sum_{n \geq 1} \frac{\sigma_{s}(n)}{n^{s / 2}} K_{s / 2}\left(\frac{2 \pi n}{x}\right) & =4 x \sum_{n \geq 1} \frac{\sigma_{s}(n)}{n^{s / 2}} K_{s / 2}(2 \pi n x) \\
& +\Lambda(s)\left(x^{1-s / 2}-x^{s / 2}\right)+\Lambda(s+1)\left(x^{1+s / 2}-x^{-s / 2}\right) .
\end{aligned}
$$


We now specialize to the first few integer values of $s$.

Corollary 2.3. - Let as usual $d(n)=\sigma_{0}(n)$ be the number of divisors of $n$. We have

$$
\gamma-\log (4 \pi x)+4 \sum_{n \geq 1} d(n) K_{0}\left(\frac{2 \pi n}{x}\right)=x\left(\gamma-\log \left(\frac{4 \pi}{x}\right)+4 \sum_{n \geq 1} d(n) K_{0}(2 \pi n x)\right) .
$$

Corollary 2.4. - We have

$$
\frac{\pi}{12 x}+\sum_{n \geq 1} \frac{\sigma(n)}{n} e^{-2 \pi n / x}=\frac{\pi}{12} x+\sum_{n \geq 1} \frac{\sigma(n)}{n} e^{-2 \pi n x}-\frac{\log (x)}{2} .
$$

Both corollaries follow quite easily from the theorem with $s=0$ and $s=1$ respectively.

This second corollary is in fact the transformation formula of the logarithm of Dedekind's eta function. More precisely:

Corollary 2.5. - Set

$$
L(x)=-\frac{\pi x}{12}+\sum_{n \geq 1} \log \left(1-e^{-2 \pi n x}\right),
$$

which is equal to $\log (\eta(i x))$. We have

$$
L\left(\frac{1}{x}\right)=L(x)+\frac{\log (x)}{2} .
$$

Corollary 2.6. -

$$
\frac{\pi}{6}(1-x)+4 x \sum_{n \geq 1} \frac{\sigma_{2}(n)}{n} K_{1}(2 \pi n x)=\frac{\zeta(3)}{2 \pi}\left(\frac{1}{x}-x^{2}\right)+4 \sum_{n \geq 1} \frac{\sigma_{2}(n)}{n} K_{1}\left(\frac{2 \pi n}{x}\right) .
$$

This is the theorem for $s=2$.

For $s \geq 3 \mathrm{odd}$, we obtain from the theorem formulas which are closely linked to the theory of Eichler-Shimura of $(k-2)$-fold integrals of modular forms of weight $k$, giving pseudo-modular forms of weight $2-k$. For instance, for $s=3$ we obtain directly:

Corollary 2.7. -

$$
\begin{aligned}
& \frac{\zeta(3)}{2 \pi}\left(\frac{1}{x}-x\right)+2 \sum_{n \geq 1} \frac{\sigma_{3}(n)}{n^{2}}\left(1+\frac{1}{2 \pi n x}\right) e^{-2 \pi n x} \\
= & \frac{\pi^{2}}{90}\left(\frac{1}{x^{2}}-x^{2}\right)+2 \sum_{n \geq 1} \frac{\sigma_{3}(n)}{n^{2}}\left(1+\frac{x}{2 \pi n}\right) e^{-2 \pi n / x} .
\end{aligned}
$$

However, as mentioned above, this is not the point. A consequence is the following:

Corollary 2.8. - Set

$$
H(x)=-\frac{\pi^{3}}{180} x^{3}-\frac{\pi^{3}}{72} x+\frac{\zeta(3)}{2}+\sum_{n \geq 1} \frac{\sigma_{3}(n)}{n^{3}} e^{-2 \pi n x} .
$$


Then $H^{\prime \prime \prime}(x)=-\left(\pi^{3} / 30\right) E_{4}(i x)$, where $E_{4}(\tau)$ is the usual holomorphic Eisenstein series of weight 4 , and $H$ satisfies the functional equation

$$
H\left(\frac{1}{x}\right)=-\frac{1}{x^{2}} H(x)
$$

in other words is a "pseudo-modular form" of weight -2 .

This is quite easily proved by integration, except that there is an a priori unknown "constant of integration", which in the above corollary is the coefficient of $x$. To compute this constant explicitly, we need to compute the asymptotic expansion of both sides, and after some work we obtain the above formula. As mentioned above, this type of formula exists for all odd integral values of $s$, with the series $\sum_{n \geq 1}\left(\sigma_{s}(n) / n^{s}\right) e^{-2 \pi n x}$ in the right-hand side.

\section{The Second Theorem and Specializations}

3.1. Integral Representations. - Note the following integral representations of the sums that we will consider:

Proposition 3.1. - For $x>0$ and all $s \in \mathbb{C}$ we have

$$
\int_{0}^{\infty} \frac{d t}{t^{s+1}\left(e^{2 \pi x t}-1\right)\left(e^{2 \pi / t}-1\right)}=2 x^{s / 2} \sum_{n \geq 1} n^{s / 2} \sigma_{-s}(n) K_{s}(4 \pi \sqrt{n x}) .
$$

Corollary 3.2. - Under the same conditions, for all $j \geq 0$ we have

$$
\int_{0}^{\infty} \frac{P_{j}\left(e^{2 \pi x t}\right)}{t^{s+1}\left(e^{2 \pi x t}-1\right)^{j+1}\left(e^{2 \pi / t}-1\right)} d t=(-1)^{j} 2 x^{s / 2} \sum_{n \geq 1} n^{s / 2+j} \sigma_{-s-j}(n) K_{s}(4 \pi \sqrt{n x}),
$$

where $P_{j}(X)$ is the sequence of polynomials defined by induction by $P_{0}(X)=1$ and $P_{j}(X)=$ $X(X-1) P_{j-1}^{\prime}(X)-j X P_{j}(X)$ for $j \geq 1$.

Corollary 3.3. - We have

$$
\int_{0}^{\infty} \frac{\log \left(1-e^{-2 \pi x t}\right)}{t^{s+1}\left(e^{2 \pi / t}-1\right)}=-2 x^{s / 2} \sum_{n \geq 1} n^{s-3 / 2} \sigma_{1-s}(n) K_{s}(4 \pi \sqrt{n x}) .
$$

These results are proved by replacing $K_{s}$ by its integral definition, and then integrating several times.

The main results of this section give formulas for the right hand side of the proposition.

3.2. The Case $s \notin \mathbb{Z}$. -

Theorem 3.4. - Let $s \notin \mathbb{Z}$ such that $\operatorname{Re}(s) \geq 0$. For any integer $k$ such that $k \geq\lfloor(\operatorname{Re}(s)+$ 1)/2」 we have the identity

$$
\begin{aligned}
& 8 \pi x^{s / 2} \sum_{n \geq 1} n^{s / 2} \sigma_{-s}(n) K_{s}(4 \pi \sqrt{n x})=A(s, x) \zeta(s)+B(s, x) \zeta(s+1) \\
& +\frac{2}{\sin (\pi s / 2)}\left(\sum_{1 \leq i \leq k} \zeta(2 i) \zeta(2 i-s) x^{2 i-1}+x^{2 k+1} \sum_{n \geq 1} \sigma_{-s}(n) \frac{n^{s-2 k}-x^{s-2 k}}{n^{2}-x^{2}}\right),
\end{aligned}
$$


where

$$
A(s, x)=\frac{x^{s-1}}{\sin (\pi s / 2)}-(2 \pi)^{1-s} \Gamma(s) \text { and } B(s, x)=(2 \pi)^{-s-1} \Gamma(s+1) \frac{2}{x}-\frac{\pi x^{s}}{\cos (\pi s / 2)} .
$$

\section{Remarks.}

(1) In the above theorem, if $x=n \in \mathbb{Z}_{\geq 1}$ the expression $\left(n^{s-2 k}-x^{s-2 k}\right) /\left(n^{2}-x^{2}\right)$ is of course to be interpreted as its limit as $x$ tends to $n$, in other words as $(s / 2-k) n^{s-2 k-2}$.

(2) The condition $\operatorname{Re}(s) \geq 0$ is not restrictive since the left hand side of the identity is invariant under the change of $s$ into $-s$.

(3) Although the theorem is valid when $k \geq\lfloor(\operatorname{Re}(s)+1) / 2\rfloor$, the convergence of the series on the right hand side is as fast as possible without acceleration only when $k \geq\lceil\operatorname{Re}(s) / 2\rceil$, in other words $k \geq\lfloor(\operatorname{Re}(s)+2) / 2\rfloor$ if $\operatorname{Re}(s) \notin 2 \mathbb{Z}$.

The proof of the theorem involves integrating the formula of the theorem of the preceding section, and doing a careful extension process to show that it is valid for all $s$ as given.

The specializations of the above theorem to $s=1 / 2$ and $s=3 / 2$ are as follows:

\section{Corollary 3.5. -}

$$
\begin{aligned}
2 \pi \sum_{n \geq 1} \sigma_{-1 / 2}(n) e^{-4 \pi \sqrt{n x}}= & 2 x \sum_{n \geq 1} \frac{\sigma_{-1 / 2}(n)}{\left(n^{1 / 2}+x^{1 / 2}\right)(n+x)} \\
& +\left(\frac{1}{4 \pi x}-\pi x^{1 / 2}\right) \zeta(3 / 2)-\left(\pi-\frac{1}{x^{1 / 2}}\right) \zeta(1 / 2) .
\end{aligned}
$$

Corollary 3.6. -

$$
\begin{aligned}
& \sum_{n \geq 1} \sigma_{-3 / 2}(n)(4 \pi \sqrt{n x}+1) e^{-4 \pi \sqrt{n x}}=-4 x^{3} \sum_{n \geq 1} \frac{\sigma_{-3 / 2}(n)}{\left(n x^{1 / 2}+x n^{1 / 2}\right)(n+x)} \\
&+\left(\frac{3}{8 \pi^{2} x}+2 \pi x^{3 / 2}\right) \zeta(5 / 2)+\left(2 x^{1 / 2}-\frac{1}{2}\right) \zeta(3 / 2)+\frac{2 \pi^{2}}{3} x \zeta(1 / 2) .
\end{aligned}
$$

3.3. The Case $s \in 2 \mathbb{Z}$. - As usual, we assume that $s \geq 0$. The result corresponding to $s=2 m$ with $m \in \mathbb{Z}_{\geq 1}$ is as follows:

Theorem 3.7. - Let $m \in \mathbb{Z}_{\geq 1}$. We have the identity

$$
\begin{aligned}
& 8(-1)^{m} \sum_{n \geq 1} \frac{\sigma_{2 m}(n)}{n^{m}} K_{2 m}(4 \pi \sqrt{n x})= \\
& \quad \frac{4}{\pi^{2}}\left(x^{m+1} \sum_{n \geq 1} \frac{\sigma_{2 m}(n)}{n^{2 m}} \frac{\log (n / x)}{n^{2}-x^{2}}-\sum_{1 \leq i \leq m-1} \zeta(2 i) \zeta^{\prime}(2 i-2 m) x^{2 i-m-1}\right) \\
& +\frac{2}{\pi^{2}} x^{m-1}\left(\zeta(2 m) \log (2 \pi x)+\zeta^{\prime}(2 m)\right)+\frac{B_{2 m}}{2 m x^{m}} \\
& +\left((-1)^{m} \frac{4(2 m) !}{(2 \pi)^{2 m+2} x^{m+1}}-x^{m}\right) \zeta(2 m+1) .
\end{aligned}
$$


Note that by the functional equation, if desired we can replace $\zeta^{\prime}(2 i-2 m)$ by its expression in terms of $\zeta(2 m+1-2 i)$.

For instance, by simple replacement, for $s=2$, in other words for $m=1$, we obtain

Corollary 3.8. - We have the identity

$$
\begin{aligned}
-8 \sum_{n \geq 1} \frac{\sigma_{2}(n)}{n} K_{2}(4 \pi \sqrt{n x})= & \frac{4}{\pi^{2}} x^{2} \sum_{n \geq 1} \frac{\sigma_{2}(n)}{n^{2}} \frac{\log (n / x)}{n^{2}-x^{2}} \\
& +\frac{1}{3}\left(\log (2 \pi x)+\frac{\zeta^{\prime}(2)}{\zeta(2)}\right)+\frac{1}{12 x}-\left(\frac{1}{2 \pi^{4} x^{2}}+x\right) \zeta(3) .
\end{aligned}
$$

The result corresponding to $s=m=0$ is as follows:

Theorem 3.9. - We have

$$
4 \sum_{n \geq 1} d(n) K_{0}(4 \pi \sqrt{n x})=\frac{2 x}{\pi^{2}} \sum_{n \geq 1} d(n) \frac{\log (n / x)}{n^{2}-x^{2}}-\left(\gamma+\frac{\log (x)}{2}+\frac{\log \left(2 \pi x^{1 / 2}\right)}{\pi^{2} x}\right) .
$$

Here again we need to do a careful limiting process.

Corollary 3.10. -

$$
\begin{array}{r}
-4 \pi^{3} x^{-1 / 2} \sum_{n \geq 1} n^{1 / 2} d(n) K_{1}(4 \pi \sqrt{n x})=\sum_{n \geq 1} d(n) \log (n / x) \frac{n^{2}+x^{2}}{\left(n^{2}-x^{2}\right)^{2}} \\
-\sum_{n \geq 1} d(n) \frac{1}{n^{2}-x^{2}}+\frac{1}{4}\left(\frac{2 \log \left(2 \pi x^{1 / 2}\right)-1}{x^{2}}-\frac{\pi^{2}}{x}\right) .
\end{array}
$$

Simply compute derivatives.

3.4. The Case $s \in 1+2 \mathbb{Z}$. - Once again we assume that $s \geq 0$. The result corresponding to $s=2 m+1$ with $m \in \mathbb{Z}_{\geq 1}$ is as follows:

Theorem 3.11. - Let $m \in \mathbb{Z}_{\geq 1}$. We have the identity

$$
\begin{aligned}
8 \pi(-1)^{m} x^{1 / 2} \sum_{n \geq 1} & \frac{\sigma_{2 m+1}(n)}{n^{m+1 / 2}} K_{2 m+1}(4 \pi \sqrt{n x})=-2 x^{m+2} \sum_{n \geq 1} \frac{\sigma_{2 m+1}(n)}{n^{2 m+2}(n+x)} \\
& +2 \sum_{1 \leq i \leq m} \zeta(2 i) \zeta(2 i-2 m-1) x^{2 i-m-1} \\
& +\left(x^{m}+(-1)^{m+1} \frac{(2 m) !}{(2 \pi)^{2 m} x^{m}}\right) \zeta(2 m+1)+\frac{B_{2 m+2}}{2(m+1) x^{m+1}} \\
& +2 x^{m+1}\left((\log (x)+\gamma) \zeta(2 m+2)+\zeta^{\prime}(2 m+2)\right)
\end{aligned}
$$

The result corresponding to $m=0$, in other words to $s=1$, is as follows: 
Theorem 3.12. - We have the identity

$$
\begin{aligned}
8 \pi x^{1 / 2} \sum_{n \geq 1} \frac{\sigma(n)}{n^{1 / 2}} K_{1}(4 \pi \sqrt{n x}) & =-2 x^{2} \sum_{n \geq 1} \frac{\sigma(n)}{n^{2}(n+x)} \\
& +\log (2 \pi x)+\gamma+\frac{1}{12 x}+2 x\left((\log (x)+\gamma) \zeta(2)+\zeta^{\prime}(2)\right),
\end{aligned}
$$

where as usual $\sigma(n)=\sigma_{1}(n)$.

Corollary 3.13. - We have the identities

$$
\begin{aligned}
8 \pi^{2} \sum_{n \geq 1} \sigma(n) K_{0}(4 \pi \sqrt{n x})= & \sum_{n \geq 1} \sigma(n)\left(\frac{1}{n^{2}}-\frac{1}{(n+x)^{2}}\right) \\
& -\frac{\pi^{2}}{6}-\frac{1}{2 x}+\frac{1}{24 x^{2}}-\left((\log (x)+\gamma) \zeta(2)+\zeta^{\prime}(2)\right) \\
8 \pi^{3} x^{1 / 2} \sum_{n \geq 1} n^{1 / 2} \sigma(n) K_{1}(4 \pi \sqrt{n x}) & =-x \sum_{n \geq 1} \frac{\sigma(n)}{(n+x)^{3}}+\frac{\pi^{2}}{12}-\frac{1}{4 x}+\frac{1}{24 x^{2}}
\end{aligned}
$$

and

$$
16 \pi^{4} \sum_{n \geq 1} n \sigma(n) K_{0}(4 \pi \sqrt{n x})=\sum_{n \geq 1} \sigma(n) \frac{n-2 x}{(n+x)^{4}}-\frac{1}{4 x^{2}}+\frac{1}{12 x^{3}} .
$$

3.5. Asymptotics of Sums $\sum_{n \geq 1} \sigma_{-m}(n) f(x / n)$. - Set $T_{m}(f)=\sum_{n \geq 1} \sigma_{-m}(n) f(x / n)$. A careful study of these sums for a large and useful class of functions $f$ is necessary, so as to obtain their asymptotic expansions as $x \rightarrow \infty$. This is quite nontrivial, and requires several pages of computations. We only give the results for the functions that we need:

Example 1. $f(t)=\log (1+t)-t$ and $m \geq 2$. In that case:

$$
\begin{aligned}
T_{m}(f)(x)= & -\zeta(m+1) x \log (x)+\left(-\zeta^{\prime}(m+1)+(1-\gamma) \zeta(m+1)\right) x \\
& -(\zeta(m) / 2) \log (x)-\zeta^{\prime}(m) / 2-(\log (2 \pi) / 2) \zeta(m)+o(1) .
\end{aligned}
$$

Example 2. $f(t)=\log (t) \log \left(\left|1-t^{2}\right|\right)$ and $m \geq 2$. In that case:

$$
\begin{aligned}
T_{m}(f)(x)= & \left(\pi^{2} / 2\right) \zeta(m+1) x-\zeta(m) \log ^{2}(x)-2\left(\zeta^{\prime}(m)+\log (2 \pi) \zeta(m)\right) \log (x) \\
& -\zeta^{\prime \prime}(m)-2 \log (2 \pi) \zeta^{\prime}(m)+2 \zeta^{\prime \prime}(0) \zeta(m)+o(1) .
\end{aligned}
$$

Example 3. $f(t)=\operatorname{Li}_{2}\left(t^{2}\right)$ and $m \geq 2$. In that case:

$$
\begin{aligned}
T_{m}(f)(x)= & \zeta(m) \log ^{2}(x)+2\left(\zeta^{\prime}(m)+\log (2 \pi) \zeta(m)\right) \log (x)+\zeta^{\prime \prime}(m) \\
& +2 \log (2 \pi) \zeta^{\prime}(m)-\left(2 \zeta^{\prime \prime}(0)+\pi^{2} / 6\right) \zeta(m)+o(1) .
\end{aligned}
$$

\section{Integration of the Formulas for $s \in \mathbb{Z}$}

We can integrate the results that we have obtained for $m \in \mathbb{Z}$. Integration term by term is trivially justified, but the whole difficulty lies in the determination of the constant of integration. This is done by comparing the asymptotic expansions of both sides, that of the right-hand side being obtained thanks to the study mentioned above, and in particular of the specific examples. 
4.1. The Case $s \in 2 \mathbb{Z}, s \neq 0$. -

Theorem 4.1. - Let $m \in \mathbb{Z}_{\geq 1}$. We have the identity

$$
\begin{aligned}
& \frac{4}{\pi} x^{-m+1 / 2}(-1)^{m-1} \sum_{n \geq 1} \frac{\sigma_{2 m}(n)}{n^{m+1 / 2}} K_{2 m-1}(4 \pi \sqrt{n x})= \\
& \quad \frac{1}{\pi^{2}}\left(\sum_{n \geq 1} \frac{\sigma_{2 m}(n)}{n^{2 m}}\left(2 \log \left(\frac{x}{n}\right) \log \left(\left|1-\frac{x^{2}}{n^{2}}\right|\right)+\operatorname{Li}_{2}\left(\frac{x^{2}}{n^{2}}\right)\right)\right. \\
& \left.\quad+4 \sum_{1 \leq i \leq m-1} \zeta(2 i) \zeta^{\prime}(2 i-2 m) \frac{x^{2 i-2 m}}{2 m-2 i}\right) \\
& +\frac{2}{\pi^{2}}\left(\left(\zeta(2 m) \log (2 \pi)+\zeta^{\prime}(2 m)\right) \log (x)+\frac{\zeta(2 m)}{2} \log ^{2}(x)\right) \\
& \quad-\frac{B_{2 m}}{2 m(2 m-1) x^{2 m-1}}+\left((-1)^{m-1} \frac{4(2 m-1) !}{(2 \pi)^{2 m+2} x^{2 m}}-x\right) \zeta(2 m+1)+C_{2 m},
\end{aligned}
$$

where $C_{2 m}$ is a constant given by

$$
C_{2 m}=\zeta^{\prime \prime}(2 m)+2 \log (2 \pi) \zeta^{\prime}(2 m)+\left(\pi^{2} / 6-2 \zeta^{\prime \prime}(0)\right) \zeta(2 m)
$$

As mentioned above, the only difficulty is in the computation of $C_{2 m}$, which is done by making $x \rightarrow \infty$ and comparing asymptotic expansions.

\subsection{The Case $s=0$. -}

\section{Theorem 4.2. -}

$$
\begin{aligned}
\frac{-2}{\pi} x^{1 / 2} & \sum_{n \geq 1} \frac{d(n)}{n^{1 / 2}} K_{1}(4 \pi \sqrt{n x}) \\
= & \frac{1}{2 \pi^{2}} \sum_{n \geq 1} d(n)\left(2 \log \left(\frac{x}{n}\right) \log \left(\left|1-\frac{x^{2}}{n^{2}}\right|\right)+\operatorname{Li}_{2}\left(\frac{x^{2}}{n^{2}}\right)\right) \\
& -\left(\left(\gamma-\frac{1}{2}\right) x+\frac{x \log (x)}{2}+\frac{\log (2 \pi)}{\pi^{2}} \log (x)+\frac{\log ^{2}(x)}{4 \pi^{2}}\right)+C_{0}
\end{aligned}
$$

where $C_{0}$ is an explicit constant which I have not had the time to compute.

4.3. The Case $s=2 m+1, m \geq 1$. - 
Theorem 4.3. -

$$
\begin{aligned}
4(-1)^{m+1} x^{-} & \sum_{n \geq 1} \frac{\sigma_{2 m+1}(n)}{n^{m+1}} K_{2 m}(4 \pi \sqrt{n x}) \\
= & 2 \sum_{n \geq 1} \frac{\sigma_{2 m+1}(n)}{n^{2 m+1}}\left(\log \left(1+\frac{x}{n}\right)-\frac{x}{n}\right) \\
& +2 \sum_{1 \leq i \leq m} \zeta(2 i) \frac{\zeta(2 i-2 m-1)}{2 i-2 m-1} x^{2 i-2 m-1} \\
& +\left(\log (x)+(-1)^{m} \frac{(2 m-1) !}{(2 \pi)^{2 m} x^{2 m}}\right) \zeta(2 m+1)-\frac{B_{2 m+2}}{(2 m+1)(2 m+2) x^{2 m+1}} \\
& +2\left(x \log (x) \zeta(2 m+2)+x\left((\gamma-1) \zeta(2 m+2)+\zeta^{\prime}(2 m+2)\right)\right)+C_{2 m+1} \\
= & -2 \sum_{n \geq 1} \frac{\log (\Gamma(1+x / n))}{n^{2 m+1}} \\
& +2 \sum_{1 \leq i \leq m} \zeta(2 i) \frac{\zeta(2 i-2 m-1)}{2 i-2 m-1} x^{2 i-2 m-1} \\
& +\left(\log (x)+(-1)^{m} \frac{(2 m-1) !}{(2 \pi)^{2 m} x^{2 m}}\right) \zeta(2 m+1)-\frac{B_{2 m+2}}{(2 m+1)(2 m+2) x^{2 m+1}} \\
& +2\left(x \log (x) \zeta(2 m+2)+x\left(-\zeta(2 m+2)+\zeta^{\prime}(2 m+2)\right)\right)+C_{2 m+1}
\end{aligned}
$$

where $C_{2 m+1}$ is a constant given by

$$
C_{2 m+1}=\zeta^{\prime}(2 m+1)+\log (2 \pi) \zeta(2 m+1) .
$$

\subsection{The Case $s=1$. -}

Theorem 4.4. - We have the identity

$$
\begin{aligned}
-2 \sum_{n \geq 1} & \frac{\sigma(n)}{n} K_{0}(4 \pi \sqrt{n x})=\sum_{n \geq 1} \frac{\sigma(n)}{n}\left(\log \left(1+\frac{x}{n}\right)-\frac{x}{n}\right) \\
& +\frac{1}{4} \log ^{2}(x)+\frac{\log (2 \pi)+\gamma}{2} \log (x)+C_{1}-\frac{1}{24 x} \\
& +\frac{\pi^{2}}{6} x \log (x)+x\left((\gamma-1) \zeta(2)+\zeta^{\prime}(2)\right)
\end{aligned}
$$

where $C_{1}$ is a constant given by

$$
\begin{aligned}
C_{1} & =\frac{5 \pi^{2}}{48}+\frac{\log ^{2}(2 \pi)}{4}+\frac{\gamma \log (2 \pi)}{2}-\frac{\gamma^{2}}{4}-\gamma_{1} \\
& =\frac{\pi^{2}}{16}-\frac{\log ^{2}(2 \pi)}{4}+\frac{\gamma \log (2 \pi)}{2}+\frac{\gamma^{2}}{4}-\zeta^{\prime \prime}(0),
\end{aligned}
$$

where $\gamma_{1}=\lim _{N \rightarrow \infty}\left(\sum_{1 \leq n \leq N} \log (n) / n-\log ^{2}(N) / 2\right)$. 
For the convenience of the reader, here are some numerical values:

$$
\begin{aligned}
\gamma_{1} & =-0.0728158454836767248605863758749013191377363383343 \cdots \\
\zeta^{\prime \prime}(0) & =-2.0063564559085848512101000267299604381989949101609 \cdots \\
C_{1} & =2.39247890056761851538254526825624242310295177862365 \cdots
\end{aligned}
$$

\section{References}

[1] B. Berndt, Y. Lee, and J. Sohn, Koshliakov's formula and Guinand's formula in Ramanujan's lost notebook, in K. Alladi (ed.), Surveys in number theory, Springer (2008), p. 21-42.

[2] H. Cohen, Number Theory vol I: Tools and Diophantine Equations, Graduate Texts in Math. 239, Springer-Verlag (2007).

[3] H. Cohen, Number Theory vol II: Analytic and Modern Tools, Graduate Texts in Math. 240, Springer-Verlag (2007).

January 17, 2010

Henri Cohen, Université Bordeaux I, Institut de Mathématiques, U.M.R. 5251 du C.N.R.S, 351 Cours de la Libération, 33405 TALENCE Cedex, FRANCE • E-mail : henri.cohen@math.u-bordeaux1.fr 